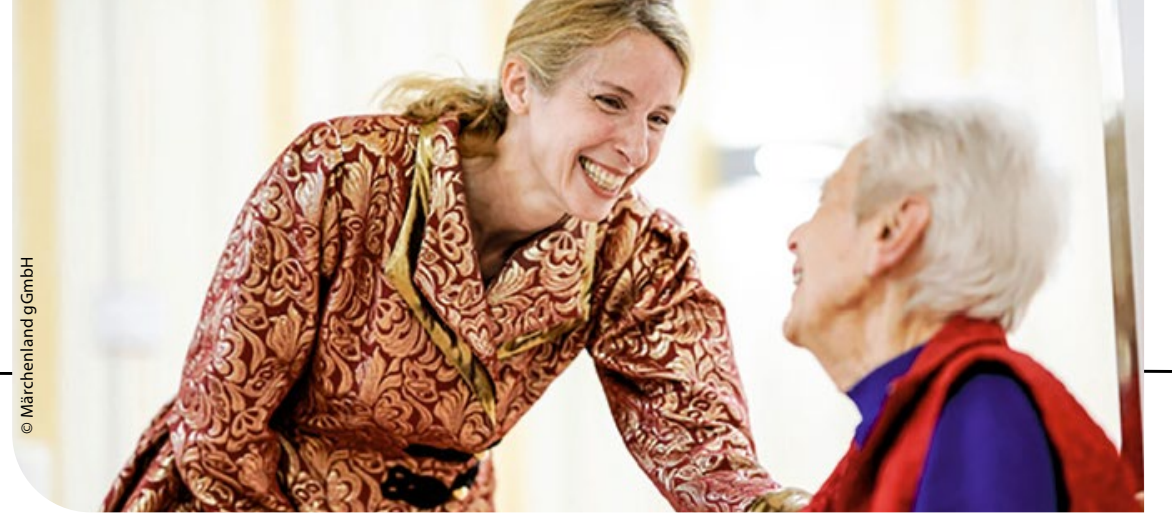

\title{
Wie wird Weihnachten 2020?
}

Weihnachten geborgen in Erinnerungen und aufgefangen in Ritualen - wie schaffen es Pflegende in diesem besonderen Jahr für ihre Bewohner die Advents-und Weihnachtszeit zu gestalten? Wir fragten in Pflegeeinrichtungen, was dort geplant ist. Eine Möglichkeit bietet auch das Projekt „Es war einmal... Märchen und Demenz" der MÄRCHENLAND - Zentrum für Prävention und Gesundheitsförderung GmbH.

Menschen mit einer dementiellen Erkrankung können mit Märchen erreicht werden. Durch das Erzählen werden eine Stärkung der kognitiven Fähigkeiten, eine Verbesserung der psychischen Gesundheit und eine soziale Interaktion begünstigt. Märchen helfen, herausforderndes Verhalten zu reduzieren und beugen auch depressiven Situationen vor. Diese Form des Einsatzes von Märchen ist eine Präventionsmaßnahme für stationäre Pflegeeinrichtungen gemäß § 5 SGB XI und der GKV nach § 20 Abs. 1 SGB V.

\section{Vielseitig einsetzbar}

Die professionellen Erzähler kommen in die jeweiligen stationären Einrichtungen. Es sollten hierfür beständige Termine vereinbart werden, damit es ein fester Bestandteil in der Wochenplanung ist. Die Erzähler begleiten Menschen, die an einer demenziellen Erkrankung leiden, indem sie wiederkehrende Rituale anbieten und das Märchen „erleben“ lassen. Dabei steht das Erzählen im Vordergrund, komplettiert durch hochwertige Arbeitsmaterialen, die in Märchenland-Boxen und in einem Märchenland-Koffer bereitgestellt werden. In den Boxen befinden sich je ein Märchenbuch, Ausmalblätter, ein Memory, eine Audio-CD und ein Märchenfilm. Diese Materialien ermöglichen weitere individuelle Tätigkeiten, wie das Interpretieren oder Ausmalen von Bildern, passende Lieder zu singen oder das Märchen von einem Zuhörenden weiter erzählen zu lassen.

Alle Erzähler sind speziell ausgebildete professionelle Demenzerzähler, die im Vorfeld über das Krankheitsbild geschult wurden sowie beispielsweise Sprachrituale beherrschen und gezielt einsetzen.

\section{Gut geschult}

In weiteren Modulen bietet die Märchenland $\mathrm{GmbH}$ Schulungen von Mitarbeitenden zu Vorlesern an. Dazu gibt es Strukturierungsgespräche per Telefon- oder Videokonferenz und Onlineschulungen. In Kooperation mit der Professorin für Pflegewissenschaft Ingrid Kollak wird das Projekt wissenschaftlich begleitet, es erfolgen Evaluation und Dokumentation vom Erzählen und den Reaktionen der Zuhörenden. 2020, das Jahr der Pandemie und der zusätzlichen Isolation von Menschen in vollstationären Pflegeeinrichtungen, bewirkt bei Menschen mit einer dementiellen Erkrankung noch mehr Unsicherheit und Angst. Viele Strukturen, die die vollstationären Pflegeeinrichtungen bisher gewährleistet haben, sowie die Einbindung von Angehörigen und Freunden können nicht mehr erbracht werden. Es ist ( $k$ )ein Zufall, zugleich auch ein Glücksfall, dass das Projekt „Es war einmal... Märchen und Demenz" als virtuelle Märchenstunde bereits vor der Pandemie geplant wurde. Dazu stellt Märchenland acht USBSticks zur Verfügung und ermöglicht, das Material über ein Online Portal zu nutzen, so dass jede teilnehmende Einrichtung virtuell die Märchenstunden gestalten kann.

Christine Schmidt-Statzkowski Pflegesachverständige maerchenunddemenz.de

\section{Gemeinsam gedrehter Gottesdienst-Film}

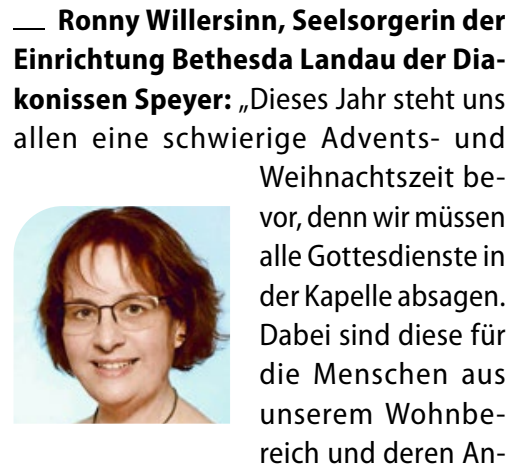

gehörige sehr wichtig. Sie werden die Nähe und das Gemeinschaftsgefühl während der Feiern vermissen. Alternativ werden wir Gottesdienste in kleineren Gruppen direkt in den Wohnbereichen feiern.

Darüber hinaus drehen wir im Vorfeld gemeinsam einen Film von einem Gottesdienst, an dem viele Bewohner und Bewohnerinnen, Mitglieder des Gottesdienst-Teams und auch Mitarbeitende mit Liedern und Gebeten in leichter Sprache beteiligt sind. Wenn sich die anderen Bewohner das Video dann an Heiligabend anschauen, werden sie vertraute Gesichter, Lieder und Verse und die vertraute Kapelle wiedererkennen.

_ Biblischer Stationenweg: In Anlehnung an unseren großen ,Biblischen Stationenweg' der vergangenen Jahre, bei dem unsere Bewohner mit großer Freude biblische Situationen nachstellten, werden wir in diesem Jahr die Weihnachtsstationen lebensgroß mithilfe von Schaufensterpuppen in unserer Kapelle gestalten. Alle Interessierten aus dem Haus können diese dann über die Feiertage in kleineren Gruppen begehen und die Weihnachtsgeschichte in leichter Sprache dazu hören." 
Personal für die Bewältigung dieser, in diesem Jahr noch größeren, belastenden Situationen ein. Wir hoffen, dass unser Personal in diesem Jahr nicht ausschließlich mit dem Screening von Besuchern beschäftigt sein wird und wir allen Bewohnern und Bewohnerinnen, Angehörigen, Mitarbeiterinnen und Mitarbeitern besinnliche Stunden im Caritas Altenzentrum St. Josef bieten können."

INTENSIV BETREUEN

\section{Viel Personal für Menschen mit Sucht}

_ Tino Gaberle, Einrichtungsleiter des Caritas-Altenzentrum St. Josef, Caritasverband Düsseldorf e.V.: „Weihnachten ist ein Fest, an dem die Familie zusammenkommt. So organisieren wir unter normalen Umständen am Heiligen Abend ein festliches Buffet für Bewohnerinnen und Bewohner sowie deren Angehörige. In

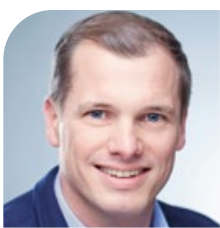
diesem Jahr wird dies leider nicht möglich sein. Neben den spirituellen Angeboten, wie Andachten und seelsorgerische Begleitung, finden die Feierlichkeiten in kleinen

Gruppen innerhalb der Wohnbereiche statt - sofern diese dann überhaupt noch zulässig sein sollten.

Weihnachten ist auch ein emotionales Fest. Gerade für Bewohnerinnen und Bewohner in Pflegeeinrichtungen geht diese Zeit mit vielen Gedanken und oft auch Trauer einher. Neben den Pflegebereichen der Einrichtung ist vor allem in unserem Suchtbereich eine intensive Betreuung der Menschen notwendig. Aufgrund der Emotionalität kann es in dieser Zeit häufiger zu Rückfällen kommen. Daher setzen wir rund um die Feiertage möglichst viel
TROTZDEM EIN FEST GESTALTEN

\section{Weihnachtsmusik am Gartenzaun}

- Beate Dickhut und Daniela Gschwind, Haus der Pflege St. Pirmin in Maikammer, Liebenau Leben im Alter gemeinnützige GmbH: „In diesem Jahr wird die Advents- und Weihnachtszeit anders sein. Corona mit den nötigen Abstand- und Hygieneregeln verhindert vieles. Dennoch werden wir auch unter diesen Rahmenbedingungen - wenn auch diesmal leider ohne Kita-Kinder - mit der sozialen Betreuung zusammen Weihnachtsgebäck backen und das Haus weihnachtlich schmücken.

In der Adventszeit werden wir zudem von Privatleuten und kleinen Vereinen musikalisch am Gartenzaun unterstützt. Unser alljährlicher Glühweinabend mit Weihnachtsgeschichten sowie ein Weihnachtswunschkonzert werden höchstwahrscheinlich stattfinden. Außerdem feiern wir, wie jeden Sonntag, gemeinsam über einen Live-Stream die Gottesdienste mit, unsere Bewohner werden wöchentlich durch besondere Grußworte des Pfarrers integriert. Unsere Weihnachtsfeier im Haus muss in diesem Jahr aber leider ohne die Angehörigen stattfinden. Wir werden trotzdem ein Fest mit gutem Essen und hauseigener Musik gestalten. An Heiligabend planen wir weiterhin die Aufführung eines Krippenspiels."
AUF JEDEN FALL ERREICHBAR

\section{Ein offenes Ohr zwischen den Jahren}

— Ingrid Haier-Radermacher, Ärztliche Leiterin der MZV Pfalzklinikum GmbH, Fachärztin für Psychiatrie und Psychotherapie, Fachärztin für Allgemeinmedizin: „Im Medizinischen Versorgungszentrum Pfalzklinikum mit seinen Zweigstellen Kaiserslautern, Kusel und Landau behandeln wir täglich viele Menschen mit psychischen und neurologischen Erkrankungen, stets unter Berück-

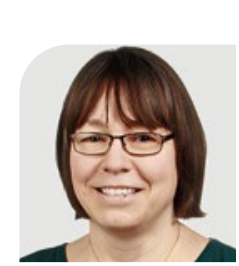
sichtigung strenger $\mathrm{Hy}$ gienemaßnahmen wegen der Corona-Pandemie.

Für unsere Klienten stellt die Vorweihnachtzeit eine besondere Herausforderung dar: Durch Stress und hohe Erwartungen an das Fest selbst. Freude und Enttäuschung liegen in den Weihnachtstagen eng beieinander. Die Corona-Pandemie erschwert die Situation. Wird gemeinsames Feiern wegen der steigenden Infektionszahlen in der bekannten Form möglich sein? Wie gehen unsere Klienten damit um, dass dieses Weihnachten ihre Erwartungen vielleicht enttäuschen wird?

_ Offene Sprechstunde für alle: Im MVZ Pfalzklinikum am Standort Kaiserslautern erwarten wir, dass uns viele Menschen gerade in der letzten Woche des Jahres spontan aufsuchen wollen. Daher sind wir zwischen Weihnachten und Neujahr flexibel und spontan ansprechbar. Für Menschen, die zu uns in die Praxisräume kommen möchten, bieten wir ganztägig eine offene Sprechstunde ein. Für die Menschen, die uns nicht aufsuchen können oder die durch Corona eingeschränkt sind, sind wir per Telefon- oder Videosprechstunde erreichbar." 